\title{
Exact solutions of the two-dimensional Boussinesq and dispersive water waves equations
}

\author{
F. P. Barrera ${ }^{1}$, T. Brugarino ${ }^{2}$ \& F. Montano ${ }^{1}$ \\ ${ }^{1}$ Dip. di Ingegneria dei Trasporti, Italy \\ ${ }^{2}$ Dip. di Metodi e Modelli Matematici Università di Palermo, \\ Facoltà d'Ingegneria Viale delle Scienze, 90128 Palermo, Italy
}

\begin{abstract}
In this paper two-dimensional Boussinesq and dispersive water waves equations are investigated in exact solutions. The Exp-function method is used for seeking exact solutions of the equations through symbolic computation.

Keywords: analytical solutions, nonlinear waves equations, Exp-function method.
\end{abstract}

\section{Introduction}

Recently new methods have been presented to solve the analytical solutions of the nonlinear wave equations, tanh-function method [1, 2], homotopy method [3] and Adomian decomposition method [4]. The Exp-function method was proposed by $\mathrm{He}$ and $\mathrm{Wu}$ to obtain solutions of nonlinear evolution equations arising in many physic problems [5]. It is simple to find numerical solutions of linear systems using computers, but this is not true for nonlinear problems. Indeed, numerical methods are connected to initial solutions and it is not easy to have convergent results for strong nonlinearity.

The procedure of the Exp-function method for the solution of PDE is straightforward. The symbol computation is an essential tool to apply the presented method.

The examination of two-dimensional Boussinesq equation arises when we consider the propagation of gravity waves on the surface of water. The structure of this equation leads to consider the propagation of waves in opposite directions. 
The dispersive long wave equations govern the propagation of long waves in shallow water. This problem consists of pair of coupled partial differential equations.

\section{Exp-function method}

We consider nonlinear partial differential equations:

$$
H\left(u, u_{x}, u_{y}, u_{t}, u_{x x}, u_{x y}, u_{y y}, \ldots\right)=0
$$

To find solutions the following transformation needs to be introduced:

$$
u(x, y, t)=u(\eta), \quad \eta=k x+h y-\omega t
$$

where $k, h$ and $\omega$ are constants and so we can convert Eq. (1) into ordinary differential equation:

$$
G\left(u, u^{\prime}, u^{\prime \prime}, u^{\prime \prime \prime}, \ldots\right)=0
$$

the prime denoting differentiation respect to $\eta$.

According to the Exp-function method, we assume that the solution of Eq. (2) can be expressed in the following form:

$$
u(\eta)=\frac{\sum_{n=-c}^{d} a_{n} \exp (n \eta)}{\sum_{m=-p}^{q} b_{m} \exp (m \eta)}
$$

In order to determine the values of $c$ and $p$, balancing the linear term of the highest order of Eq. (1) with the highest order nonlinear term, we obtain:

$$
d=c
$$

Similarly, to determine the values of $d$ and $q$ we balance the linear term of the lowest order of Eq. (1) with the lowest order nonlinear term. So we obtain:

$$
q=p
$$

Substituting Eq. (3) into Eq. (2) we have a system of algebraic equations for $a_{n}$ and $b_{m}$.

Solving this system using MATHEMATICA we carry out the values of the coefficients. 


\section{Two-dimensional Boussinesq equation}

We consider the two-dimensional Boussinesq equation:

$$
v_{t t}-v_{x x}+3\left(v^{2}\right)_{x x}-v_{x x x x}-v_{y y}=0
$$

This equation describes the propagation of waves in opposite directions in $(2+1)$ dimensions [6]. Using the wave variable:

$$
\eta=k x+h y-\omega t
$$

it is possible to lead Eq. (4) to an ordinary differential equation:

$$
\left(\omega^{2}-k^{2}-h^{2}\right) v^{\prime \prime}+3 k^{2}\left(v^{2}\right)^{\prime \prime}-k^{4} v^{(i v)}=0
$$

According to the Exp-function method, we assume that the solution of Eq. (5) can be written as follows:

$$
v(\eta)=\frac{a_{1} \exp (\eta)+a_{0}+a_{-1} \exp (-\eta)}{b_{2} \exp (2 \eta)+b_{1} \exp (\eta)+b_{0}+b_{-1} \exp (-\eta)+b_{-2} \exp (-2 \eta)}
$$

We have set $c=1$ and $q=2$. Without compromising the generality we can assume $a_{-1} \neq 0$ and Eq. (6) can be simplified as

$$
v(\eta)=\frac{a_{1} \exp (\eta)+a_{0}+\exp (-\eta)}{b_{2} \exp (2 \eta)+b_{1} \exp (\eta)+b_{0}+b_{-1} \exp (-\eta)+b_{-2} \exp (-2 \eta)}
$$

Substituting Eq. (7) into Eq. (5) and equating to zero the coefficients of all powers of $\exp (n \eta)$ we find a set of algebraic equations for $a_{0}, a_{1}, b_{2}, b_{1}, b_{0}, b_{-1}$, $b_{-2}$. Solving this system we obtain

$$
\omega^{2}=h^{2}+k^{2}+k^{4}
$$

and the following coefficients:

$$
a_{0}=-2 k^{2} b_{0}, a_{1}=k^{4} b_{0}^{2}, b_{1}=b_{-1}=0, b_{2}=\frac{a_{1}^{2}}{k^{2} a_{0}}, b_{-2}=\frac{1}{k^{2} a_{0}}
$$

Substituting $\omega$ and the coefficients into Eq. (7), we obtain the following exact solution of the two-dimensional Boussinesq equation

$$
v(x, y, t)=-\frac{2 k^{4} b_{0} \exp \left(k x+h y-t \sqrt{h^{2}+k^{2}+k^{4}}\right)}{\left(1+k^{2} b_{0} \exp \left(k x+h y-t \sqrt{h^{2}+k^{2}+k^{4}}\right)\right)^{2}}
$$




\section{Dispersive water waves equations}

The problem of the propagation of long waves in shallow water is governed by a pair of coupled nonlinear partial differential equations [7]:

$$
\left\{\begin{array}{l}
u_{t}=u u_{x}+h_{x}+\theta u_{x x} \\
h_{t}=(h u)_{x}+\theta h_{x x}
\end{array}\right.
$$

Using the variable:

$$
\eta=k x-\omega t
$$

it is possible to lead Eq.s (8) to a couple of nonlinear ordinary differential equations:

$$
\left\{\begin{array}{l}
\omega u_{\eta}+k u u_{\eta}+k h_{\eta}+k^{2} \theta u_{\eta \eta}=0 \\
\omega h_{\eta}+k(h u)_{\eta}+k^{2} \theta h_{\eta \eta}=0
\end{array}\right.
$$

Following the Exp-function method, we set:

$$
u(\eta)=\frac{a_{1} \exp (\eta)+a_{0}+a_{-1} \exp (-\eta)}{b_{2} \exp (2 \eta)+b_{1} \exp (\eta)+b_{0}+b_{-1} \exp (-\eta)+b_{-2} \exp (-2 \eta)}
$$

and

$$
h(\eta)=\frac{c_{1} \exp (\eta)+c_{0}+c_{-1} \exp (-\eta)}{d_{2} \exp (2 \eta)+d_{1} \exp (\eta)+d_{0}+d_{-1} \exp (-\eta)+d_{-2} \exp (-2 \eta)}
$$

Substituting Eq. (10) and Eq. (11) into Eq. (9) we obtain

$$
\omega=k^{2}
$$

and, solving the system, for dispersive water waves equations, we find:

$$
u(x, t)=-\frac{2 k \theta \exp (k x)}{\exp (k x)-b_{-2} \exp \left(k^{2} \theta t\right)}
$$

and

$$
h(x, t)=-\frac{4 k^{2} \theta^{2} b_{-2} \exp (k(x+k \theta t))}{\left(\exp (k x)-b_{-2} \exp \left(k^{2} \theta t\right)\right)^{2}}
$$

\section{Conclusions}

In this paper we find exact solutions of two-dimensional Boussinesq equation and of the dispersive water waves equations using a simple and direct method called the Exp-function method. This is a straight and promising tool to solve non linear evolution equations arising in physics and engineering.

But, even if this scenario could be straightforward from one hand, the implementation of it is a difficult task, mainly for the amount of calculations.

In future we propose to solve the massive system of algebraic equations given by the Exp-function method using the method Gröbner basis of non linear algebra and numerical methods. 


\section{References}

[1] L. Huibin and W. Kelin 1990 J. Phys. A 23 (1990).

[2] W. Malfliet Am. J. Phys. 60 (1992).

[3] S. Liao Appl. Math. Comput. 147 (2004).

[4] M. Dehghan and M. Tatari Phys. Scr. 73 (2006).

[5] J. H. He and X. H. Wu Chaos, Soliton and Fractals 30 (2006).

[6] R. S. Johnson J. Fluid Mech. 323 (1996).

[7] B. A. Kupershmidt Comm. Math. Phys. 99 (1985). 